\title{
The symptom burden of autonomic dysfunction is positively associated with chronic rhinosinusitis status*
}

\author{
W.C. Chen', Y.T. Chang'2, S.F. Chen², W.C. Lin³, Y.Y. Su', S.D. Luo' \\ ' Department of Otolaryngology, Kaohsiung Chang Gung Memorial Hospital and Chang Gung University College of \\ Medicine, Kaohsiung, Taiwan \\ 2 Department of Neurology, Cognition and Aging Centre, Kaohsiung Chang Gung Memorial Hospital and Chang Gung \\ University College of Medicine, Kaohsiung, Taiwan \\ ${ }^{3}$ Department of Diagnostic Radiology, Kaohsiung Chang Gung Memorial Hospital and Chang Gung University College \\ of Medicine, Kaohsiung, Taiwan
}

Rhinology 56: 3, 227-233, 2018

https://doi.org/10.4193/Rhin18.005

*Received for publication:

January 7, 2018

Accepted: February 27, 2018

Background: Dysregulation of the autonomic system can affect sinonasal physiological function and may exacerbate the symptom burden associated with rhinosinusitis. However, the association between autonomic dysfunction and chronic rhinosinusitis (CRS) has seldom been studied. Here, we investigated the relationship between autonomic dysfunction and CRS.

Methods: Patients with CRS who failed medical treatment were prospectively enrolled. All patients underwent pre-operative examinations and completed questionnaires, including the reflux symptom index (RSI) and the Sino-nasal Outcome Test-22 (SNOT22). Autonomic dysfunction was scored using the 31-item Composite Autonomic Symptom Score (COMPASS 31), a validated simple instrument used to evaluate dysautonomia.

Results: We prospectively enrolled a total of 89 CRS patients, including 37 with polyps (CRSwNP) and 52 without polyps (CRSsNP). The most common dysautonomic symptoms were dry eye, dry mouth, postural dizziness, and a sensation of excessive fullness after meals. Significant positive correlations were evident between COMPASS 31 and SNOT-22 scores in CRSwNP patients. CRSassociated symptoms, including cough, post-nasal drip, sleep, and psychological dysfunction, were correlated with the level of autonomic dysfunction.

Conclusions: We found a positive correlation between the symptom burdens of autonomic dysfunction and CRSwNP. The relationship between autonomic dysfunction and CRS is highly complex; further work is needed.

Key words: COMPASS 31, dysautonomia, autonomic nervous system, SNOT-22, RSI

\section{Introduction}

The autonomic nervous system (ANS), composed of the sensory, sympathetic, and parasympathetic subsystems, regulates microvascular permeability, glandular function, inflammatory cell recruitment, and subsequent activation of inflammatory responses of the airway mucosa ${ }^{(1)}$. Maintenance of a delicate balance between the operations of these systems is important to protect the upper airway and to ensure optimal physiological function. ANS dysfunction or dysregulation is involved in the pathogenesis of airway disease ${ }^{(2,3)}$. In patients with sinonasal disease, vasomotor rhinitis initially received most attention ${ }^{(4)}$. This disease, classified as non-allergic rhinitis, is perhaps associated with ANS dysfunction, in which hypoactive sympathetic tone triggering parasympathetic hyperactivity has been suggested ${ }^{(5,6)}$. In recent decades, allergic rhinitis patients have been shown to exhibit ANS dysregulation associated with parasympathetic hyperactivity and sympathetic hypoactivity ${ }^{(7,8)}$. Cerejeira et al. found that a7nAChR protein levels were elevated in chronic rhinosinusitis (CRS) patients, suggestive of a hyperactive parasympathetic anti-inflammatory reflex ${ }^{(9)}$. However, the relationships between ANS dysfunction and CRS have not been well studied.

ANS function can be assessed using a battery of ANS tests that explore cardiovagal, vasomotor adrenergic, and sudomotor 
functions ${ }^{(10)}$. However, such tests must be performed by an expert in a dedicated ANS testing laboratory under controlled conditions, and are thus not easily available. The Composite Autonomic Symptom Score (COMPASS), an index developed from the Autonomic Symptom Profile, is a well-established questionnaire that is used to evaluate the severity and distribution of dysautonomia symptoms ${ }^{(11)}$. Because the initial scoring algorithm was very complicated, and answering all 72 questions was timeconsuming, Sletten et al. developed and validated a refined and abbreviated version, COMPASS 31, for routine clinical use

(12). COMPASS 31 includes 31 questions in six domains, allowing quantitation of ANS symptoms, and automatically generates a symptom score between 0 and 100 after weighting. COMPASS 31 has been used to evaluate dysautonomic symptoms in patients with diabetic autonomic neuropathy, postural tachycardia syndrome, multiple sclerosis, and small-fiber polyneuropathy ${ }^{113}$ 14).

Theoretically, ANS dysfunction can trigger dysregulation of physiological sinonasal function and increase CRS severity. However, few studies have focused on the association between ANS dysfunction and CRS. Therefore, we formally assessed the symptoms of ANS dysfunction in patients with CRS using the new, quantitative, simplified COMPASS 31. We explored the relationships among ANS dysfunction, clinical characteristics, and the symptom burden of CRS patients, and identified particular symptoms of CRS patients exhibiting ANS dysfunction.

\section{Materials and methods}

This prospective study included patients diagnosed with CRS with polyps (CRSwNP) or CRS without polyps (CRSsNP) between May 2016 and May 2017 at the Chang Gung Memorial Hospital, Kaohsiung, Taiwan. The diagnosis criteria were those of the clinical practice guidelines of AAO-HNS (2015) and EPOS $(2012)^{(15,16)}$. All patients underwent medical treatment including intranasal corticosteroids for at least 2 months and oral steroids (depending on their condition) for 2 weeks in CRSwNP patients. Patients with residual symptoms who consented to surgical intervention were candidates for this study, and were included if they gave written informed consent. Sinonasal endoscopy and paranasal sinus computed tomography (CT) were performed, and levels of total $\lg \mathrm{E}$ and allergen-specific $\lg \mathrm{E}$ were measured before surgery. All patients completed the Sino-nasal Outcome Test-22 (SNOT-22) questionnaire, the reflux symptom index (RSI), and the COMPASS 31 questionnaire. The study protocol was approved by the medical ethics and human clinical trial committees of Chang Gung Memorial Hospital (Refs: 104-7185B, 105-3651C, 106-0270C).

Research instruments

SNOT-22
The SNOT-22 is employed globally to evaluate 22 sinonasal CRS symptoms, each scored from 0 (no problem) to 5 (problem as bad as it can be). The total scores range from 0-110, with higher scores reflecting greater disease severity ${ }^{(17)}$. SNOT-22 features five domains exploring rhinological, extranasal rhinological, and ear/facial symptoms, and psychological and sleep dysfunctions (18).

\section{COMPASS-31}

The 31-item COMPASS-31 is divided into six domains of ANS function: orthostatic intolerance (4 items); and vasomotor (3 items), secretomotor (4 items), gastrointestinal (12 items), bladder (3 items), and pupillomotor (5 items) symptoms, yielding a maximum raw score of 75 . The domain scores are then weighted using published values to generate scores ranging from 0-100, with higher scores reflecting greater ANS dysfunction ${ }^{(12)}$.

RSI

The RSI evaluates laryngopharyngeal reflux (LPR) and includes 9 items. Each item is scored from 0 (no problem) to 5 (severe problem) and the maximum score is 45 . It has been suggested that a score $\geq 13$ reflects LPR-positivity ${ }^{(19)}$.

\section{CT and nasal endoscopy}

All patients underwent high-resolution paranasal sinus CT (1-mm slices). All images were scored by the consensus of radiologist Dr L.W.C and otolaryngologist Dr C.W.C, using the Lund-Mackay system. Total scores ranged from 0-24, with higher scores indicating greater disease severity ${ }^{(20)}$. All patients also underwent endoscopic sinonasal evaluation and the findings were scored by the consensus of the otolaryngologist Dr C.W.C and Dr L.S.D using the modified Lund-Kennedy system. Total scores ranged from 0-12, with higher scores indicating greater disease severity ${ }^{(21)}$.

\section{Statistical analysis}

The two-sided Spearman rank correlation test was used to explore associations among COMPASS 31, SNOT-22, and RSI scores. A correlation $>0.5$ was considered high. The WilcoxonMann-Whitney test was used to compare data of the CRSsNP and CRSwNP groups. The chi-squared test was used for all other between-group comparisons. A p-value $<0.05$ was considered to indicate statistical significance. All analyses were performed with the aid of SPSS version 22 software (IBM Corp., Armonk, NY, USA).

\section{Results}

In total, 154 consecutive patients with CRS were initially identified. Those with diabetes mellitus, hypertension, any psychological disorder, chronic renal insufficiency, or a history of a malignant tumour were excluded. Finally, 89 patients (42 males, 


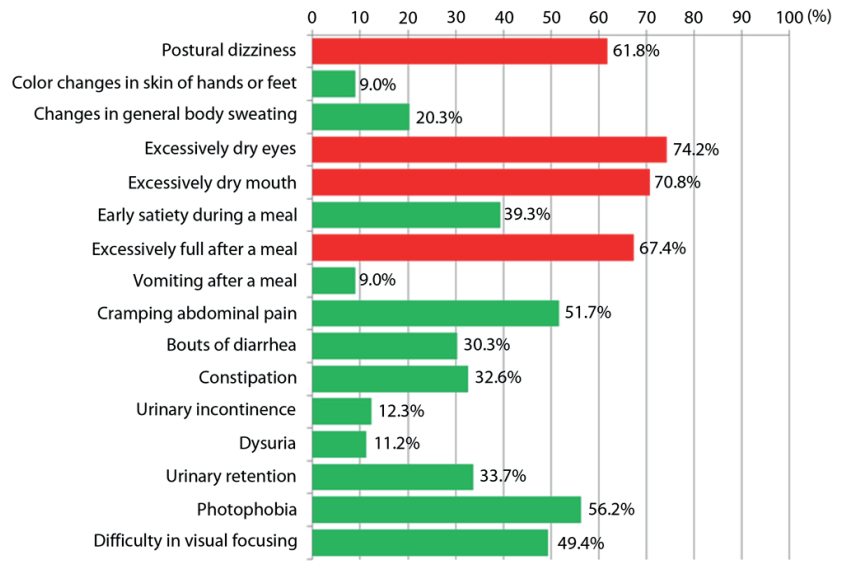

Figure 1. The prevalence of dysautonomic symptoms in chronic rhinosinusitis patients. The red bars represent the most common symptoms.

47 females; mean age 43.2 \pm 14.7 years) were prospectively enrolled. The mean COMPASS 31, SNOT-22, and RSI scores were 22.86, 44.65 , and 12.71, respectively. The most common ANS dysfunction symptoms were excessively dry eyes (74.2\%), excessively dry mouth (70.8\%), postural dizziness (61.8\%), and a feeling of excessive fullness after meals (67.4\%) (Figure 1). The CRSsNP group contained 52 patients and the CRSwNP group contained 37. The baseline characteristics of the groups did not differ significantly except for higher Lund-Mackay CT and modified Lund-Kennedy scores in the CRSwNP group (Table 1).

First, we noted that the similarity of questions among the questionnaires may contribute to a positive correlation. The symptom "dizziness" of SNOT-22 is similar to the "orthostatic intolerance" of COMPASS-31. Therefore, these scores were removed when correlation analysis. A significant positive correlation was evident between total COMPASS 31 and SNOT-22 scores ( $R$ $=0.359, P=0.001)$ in all CRS patients, and a strong correlation was found, especially in the CRSwNP group $(R=0.587, P<0.001)$ (Table 2). This positive correlation was also apparent on linear regression analysis (Figure 2). We found only marginally significant correlation ( $\mathrm{R}=0.252, \mathrm{P}=0.072$ ) in CRSsNP patients (Table 2).

On subdomain analysis, the extranasal rhinological symptom $(R=0.514, P=0.001)$, sleep dysfunction $(R=0.600, P<0.001)$, and psychological dysfunction $(\mathrm{R}=0.566, \mathrm{P}<0.001)$ SNOT-22 scores were strongly correlated with the COMPASS 31 scores of CRSwNP patients. In terms of extranasal rhinological symptoms, cough $(R=0.561, P<0.001)$ and post-nasal drip $(R=0.426, P=$ 0.008 ) were the two most important symptoms associated with ANS dysfunction (Table 3). In CRSsNP patients, no COMPASS 31 score was correlated with any SNOT-22 subdomain score.

A significant positive correlation was evident between the total
Table 1. Comparison of baseline characteristics of study group.

\begin{tabular}{|lcccc}
\multicolumn{1}{|c}{ Variables } & $\begin{array}{c}\text { Mean } \pm \text { SD } \\
(\mathbf{n}=\mathbf{8 9})\end{array}$ & $\begin{array}{c}\text { CRSsNP } \\
(\mathbf{n}=\mathbf{5 2})\end{array}$ & $\begin{array}{c}\text { CRSwNP } \\
(\mathbf{n}=\mathbf{3 7})\end{array}$ & $\mathbf{P}$ \\
\hline Age & $43.2 \pm 14.7$ & $45.5 \pm 13.6$ & $40.0 \pm 15.7$ & 0.099 \\
Gender & & & & \\
\hline Females & $47(52.8 \%)$ & $29(55.8 \%)$ & $18(48.6 \%)$ & 0.526 \\
\hline Males & $42(47.2 \%)$ & $23(44.2 \%)$ & $19(51.4 \%)$ & \\
\hline Allergic rhinitis & $36(40.4 \%)$ & $20(38.5 \%)$ & $16(43.2 \%)$ & 0.668 \\
\hline Asthma & $7(7.9 \%)$ & $3(5.8 \%)$ & $4(10.8 \%)$ & 0.443 \\
\hline Current Smoker & $19(21.3 \%)$ & $10(19.2 \%)$ & $9(24.3 \%)$ & 0.607 \\
\hline Lund-Mackay & $8.93 \pm 6.28$ & $6.21 \pm 4.61$ & $12.76 \pm 6.37$ & $<0.001$ \\
score & $4.56 \pm 3.08$ & $2.85 \pm 1.95$ & $6.97 \pm 2.75$ & $<0.001$ \\
\hline MLK score & 22.86 & 24.73 & 20.24 & 0.151 \\
\hline COMPASS 31 & \pm 13.74 & \pm 14.57 & \pm 12.18 & \\
score & 44.65 & 44.71 & 44.57 & 0.947 \\
\hline SNOT-22 score & \pm 23.16 & \pm 23.63 & \pm 22.81 & \\
\hline RSI score & $12.71 \pm 9.02$ & $13.06 \pm 9.30$ & $12.22 \pm 8.71$ & 0.665 \\
\hline
\end{tabular}

CRSsNP = Chronic rhinosinusitis without polyp; $C R S W N P=$ Chronic rhinosinusitis with polyp; MLK score = Modified Lund-Kennedy score; COMPASS 31 = 31-item Composite Autonomic Symptom Score; SNOT-22 = 22-item Sino-Nasal Outcome Test; RSI = Reflux Symptom Index.

RSI and SNOT-22 scores $(R=0.482, P<0.001)$, especially in the CRSsNP group $(R=0.576, P<0.001)$; a moderate correlation was apparent in the CRSwNP group $(\mathrm{R}=0.345, \mathrm{P}=0.037)$ (Table 2$)$. The RSI score was correlated significantly with the COMPASS 31 score in the CRSsNP group $(R=0.343, P=0.013)$; the highest COMPASS 31 score was evident in CRSsNP patients with LPR (RSI $\geq 13$ ) (Figure 3).

\section{Discussion}

The ANS modulates the inflammatory, secretory, and vascular status of the airway mucosa, and ANS dysfunction is evident in those with various airway diseases ${ }^{(3,5,22-24)}$. However, few studies have explored the association between ANS dysfunction and CRS. We explored the relationship between the symptom burden of ANS dysfunction and CRS. The mean COMPASS 31 score (22.86) of CRS patients was much higher than those of healthy controls, (8.06 and 13.34 in previous reports) ${ }^{(25,26)}$, implying a higher prevalence of ANS dysfunction in CRS patients. We found positive correlations between ANS dysfunction and CRS-related symptoms; these were strong in CRSwNP patients but marginally significant in CRSsNP patients. In the latter group, the associations may have been influenced by other factors; we found a strong correlation between CRS and LPR (Table 2). Theoretically, ANS dysregulation may exacerbate airway inflammation. However, we found no association between ANS dysfunction and disease severity as evaluated by either Lund-Mackay or mo- 


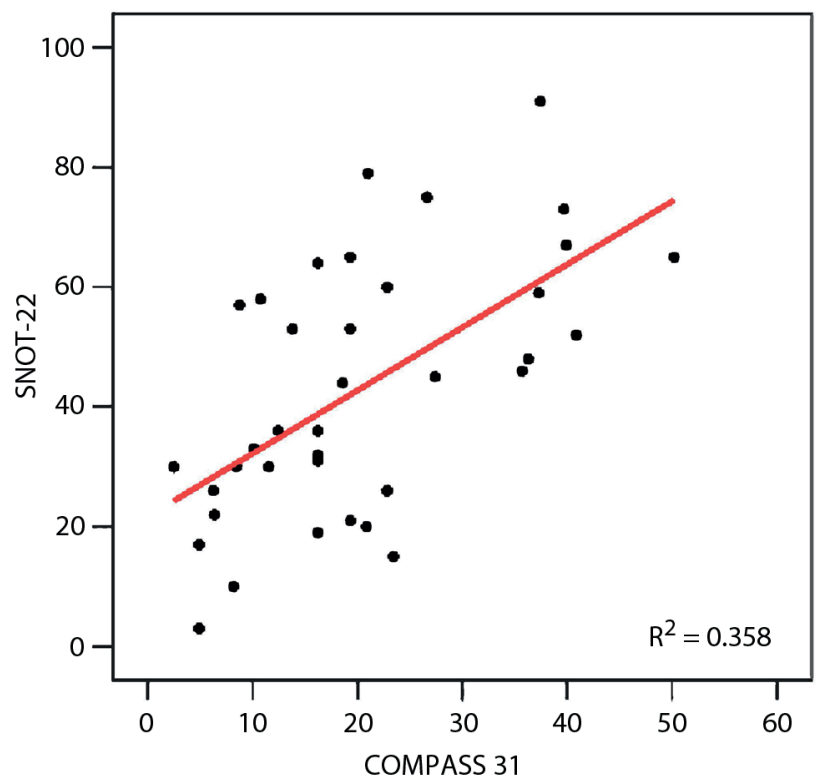

Figure 2. A positive linear regression line was apparent between the COMPASS 31 and SNOT-22 scores in CRSWNP patients; the coefficient of determination was 0.358. COMPASS $31=31$-item Composite Autonomic Symptom Score; SNOT-22 = 22-item Sino-Nasal Outcome Test.

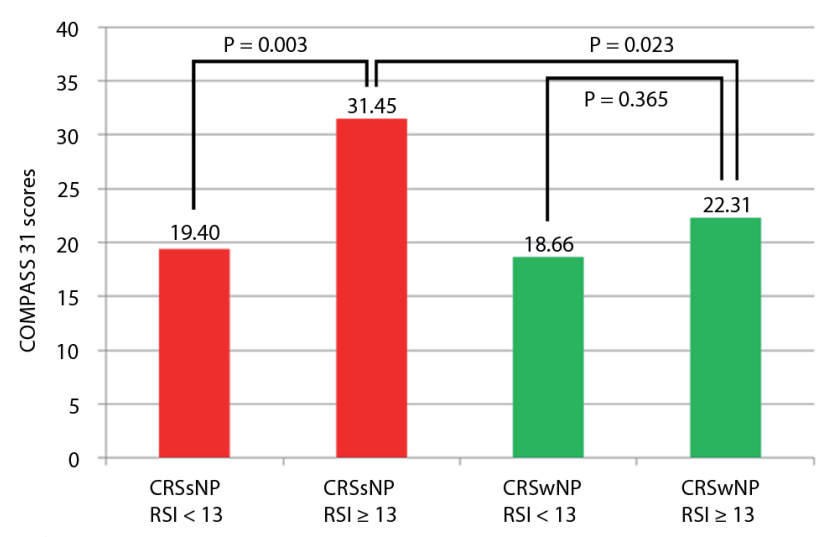

Figure 3. CRSsNP patients with LPR $(R S I \geq 13)$ had the highest COMPASS 31 scores compared with those of the other groups shown. CRSsNP = Chronic rhinosinusitis without polyp; CRSwNP = Chronic rhinosinusitis with polyp; COMPASS $31=31$-item Composite Autonomic Symptom Score; RSI = Reflux Symptom Index.

dified Lund-Kennedy scoring (Table 2). The association between rhinosinusitis and ANS dysfunction is complex, and may not be fully explained by inflammatory status alone.

The COMPASS 31 orthostatic intolerance and secretomotor domains were significantly correlated with the SNOT-22 scores of the CRSwNP group (Table 3). The orthostatic intolerance domain of COMPASS 31 evaluates principally the severity of postural dizziness, and the secretomotor domain explores sweating, dry eye, and dry mouth (symptoms of ANS dysfunction). In CRS
Table 2. Bivariate analysis of questionnaires and clinical measurements.

\begin{tabular}{|c|c|c|c|c|}
\hline & COMPASS 31 & $\mathbf{P}$ & SNOT-22 & $\mathbf{P}$ \\
\hline \multicolumn{5}{|l|}{ CRS $(n=89)$} \\
\hline SNOT-22 & $0.359+$ & 0.001 & & \\
\hline RSI & 0.288 & 0.006 & 0.482 & $<0.001$ \\
\hline $\begin{array}{l}\text { Lund-Mackay } \\
\text { score }\end{array}$ & -0.102 & 0.340 & 0.121 & 0.261 \\
\hline MLK score & -0.071 & 0.509 & 0.132 & 0.219 \\
\hline \multicolumn{5}{|c|}{ CRSsNP $(n=52)$} \\
\hline SNOT-22 & $0.252 \dagger$ & 0.072 & & \\
\hline RSI & 0.343 & 0.013 & 0.576 & $<0.001$ \\
\hline \multicolumn{5}{|c|}{ CRSwNP (n = 37) } \\
\hline SNOT-22 & $0.587+$ & $<0.001$ & & \\
\hline RSI & 0.214 & 0.204 & 0.345 & 0.037 \\
\hline
\end{tabular}

† Removal of score of dizziness from SNOT-22 when analysis. MLK score = Modified Lund-Kennedy score; CRSsNP = Chronic rhinosinusitis without polyp; CRSwNP = Chronic rhinosinusitis with polyp; COMPASS 31 = 31-item Composite Autonomic Symptom Score; SNOT-22 = 22-item Sino-Nasal Outcome Test; RSI = Reflux Symptom Index.

patients, inflamed mucosa may trigger abnormal trigeminal reflexes via the sphenopalatine ganglion, stimulating the labyrinth and dysregulating the nasal-ocular reflex ${ }^{(27,28)}$. Rhinosinusitisassociated dizziness, also termed sinugenic vertigo, has been reported ${ }^{(27)}$. Sicca symptoms (dry eye and dry mouth) were also associated with CRS in a community study conducted by Lester et al. ${ }^{(29)}$. Here, we found that $57 \%$ of all CRS patients reported both excessive dry eye and dry mouth symptoms, which have not previously been well recognized. The gastrointestinal (GI) domain scores of COMPASS 31 were also significantly correlated with the SNOT-22 scores of both CRSsNP $(R=0.340, P=0.014)$ and CRSwNP ( $R=0.367, P=0.025)$ patients. The GI COMPASS 31 domain contains questions that evaluate early satiety during a meal, excessive fullness after a meal, abdominal pain, diarrhea, and constipation. A sensation of excessive fullness after meals was the most common Gl complaint ( $67 \%$ of patients). Growing evidence indicates that gastroesophageal reflux disease (GERD)

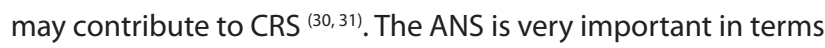
of regulation of both the $\mathrm{Gl}$ tract and the sinonasal region, and ANS distortion is evident in patients with GERD ${ }^{(30,32)}$. Lodi et al. described a hypervagal response in asthmatics with GERD. (33). Loehrl et al. found significantly more ANS dysfunction in patients with both vasomotor rhinitis and GERD than in those with vasomotor rhinitis alone ${ }^{(6)}$. Similar results were also showed in our study that CRSsNP patients with LPR had the highest COMPASS 31 score (Figure 3). 
Table 3. Correlation coefficients between COMPASS 31 and SNOT-22 in CRSwNP.

\begin{tabular}{|c|c|c|c|c|c|c|}
\hline \multirow[b]{2}{*}{ SNOT-22 } & \multicolumn{6}{|c|}{ COMPASS-31 } \\
\hline & $\begin{array}{l}\text { Orthostatic } \\
\text { intolerance }\end{array}$ & Secreto- motor & $\begin{array}{c}\text { Gastro- } \\
\text { intestinal }\end{array}$ & Bladder & Pupillo-motor & Total scores \\
\hline Need to blow nose & 0.048 & 0.240 & 0.172 & 0.175 & 0.125 & 0.150 \\
\hline Sneezing & 0.163 & $0.363^{*}$ & $0.432^{* *}$ & 0.292 & $0.332^{*}$ & $0.361^{*}$ \\
\hline Runny nose & 0.006 & 0.266 & 0.026 & -0.108 & 0.005 & 0.090 \\
\hline Blockage/ congestion of nose & 0.112 & 0.125 & 0.222 & -0.194 & -0.156 & 0.195 \\
\hline Sense of taste/smell & -0.048 & 0.134 & -0.038 & 0.192 & 0.131 & 0.001 \\
\hline Cough & $0.470^{* *}$ & $0.504^{* *}$ & 0.260 & 0.087 & 0.180 & $0.561^{* *}$ \\
\hline Post nasal discharge & $0.409^{*}$ & $0.426^{* *}$ & -0.042 & 0.179 & 0.168 & $0.426^{* *}$ \\
\hline Thick nasal discharge & 0.062 & 0.248 & 0.078 & -0.020 & 0.031 & 0.176 \\
\hline Ear fullness & 0.271 & 0.208 & 0.215 & 0.142 & 0.187 & $0.330^{*}$ \\
\hline Dizziness & $0.500^{* *}$ & $0.372^{*}$ & $0.344^{*}$ & 0.126 & 0.273 & $0.602^{* *}$ \\
\hline Ear pain/pressure & 0.343 & 0.294 & 0.274 & 0.172 & 0.186 & $0.441^{*}$ \\
\hline Facial pain/pressure & 0.194 & 0.233 & 0.319 & -0.031 & 0.206 & $0.345^{*}$ \\
\hline Difficulty falling asleep & 0.306 & 0.176 & 0.279 & -0.057 & 0.203 & $0.338^{*}$ \\
\hline Waking up at night & $0.558^{* *}$ & $0.327^{*}$ & $0.440^{* *}$ & 0.028 & 0.199 & $0.665^{* *}$ \\
\hline Lack of a good night's sleep & $0.370^{*}$ & 0.194 & $0.349^{*}$ & -0.135 & -0.031 & $0.431^{* *}$ \\
\hline Waking up tired & $0.394^{*}$ & $0.336^{*}$ & $0.409 *$ & 0.081 & 0.186 & $0.504^{* *}$ \\
\hline Fatigue during the day & $0.418^{*}$ & $0.369^{*}$ & $0.352^{*}$ & 0.175 & 0.204 & $0.510^{* *}$ \\
\hline Reduced productivity & $0.339^{*}$ & 0.277 & 0.304 & 0.130 & 0.317 & $0.473^{* *}$ \\
\hline Reduced concentration & $0.405^{*}$ & 0.251 & 0.291 & 0.075 & 0.284 & $0.501^{* *}$ \\
\hline Frustrated/Restless/Irritable & $0.399 *$ & $0.330^{*}$ & $0.373^{*}$ & 0.017 & 0.201 & $0.528^{* *}$ \\
\hline Sad & $0.435^{* *}$ & $0.336^{*}$ & 0.251 & 0.062 & 0.175 & $0.520^{* *}$ \\
\hline Embarrassed & 0.229 & 0.245 & 0.269 & 0.131 & $0.358^{*}$ & $0.356^{*}$ \\
\hline Total scores & $0.430 十^{* *}$ & $0.462^{* *}$ & $0.367^{*}$ & 0.141 & 0.266 & $0.587 \dagger^{* *}$ \\
\hline
\end{tabular}

${ }^{*} \mathrm{P}<0.05,{ }^{* *} \mathrm{P}<0.01$. + Removal of score of dizziness from SNOT-22 when analysis. CRSwNP = Chronic rhinosinusitis with polyp; COMPASS $31=$ 31-item Composite Autonomic Symptom Score; SNOT-22 = 22-item Sino-Nasal Outcome Test.

The SNOT-22 revealed that cough and post-nasal drip were the rhinological related symptoms that were correlated significantly with autonomic dysfunction in CRSwNP patients. Bucca et al. hypothesized that bronchial hyper-responsiveness caused by dysregulation of the pharyngo- or naso-bronchial reflex, and excessive nasal discharge triggered by inflammation, may activate the cough reflex ${ }^{(34)}$. Sleep and psychological dysfunctions are also correlated significantly with ANS dysfunction. Two previous meta-analyses described impaired vagal function in patients with depression or anxiety, even in medication-free patients ${ }^{35}$, ${ }^{36)}$. In a review, Miglis described increased sympathetic tone in sleep-deprived patients, regardless of their underlying disease etiologies ${ }^{(37)}$. CRS-related comorbidities and stress may contribute to ANS dysfunction or dysregulation.

The association of CRS with ANS dysfunction has seldom been investigated. To the best of our knowledge, this is the first study to explore the correlations between the symptom burden of ANS dysfunction and CRS. A strength of our study is that the work was prospective in nature; we excluded patients with systemic diseases that could possibly influence ANS function. We enrolled only patients scheduled for surgical intervention because troublesome symptoms persisted even after medical treatment. We found that the symptom burden of CRSWNP was exacerbated, particularly in terms of extranasal symptoms (cough, post-nasal drip, sleep disturbance, and psychological dysfunction) when autonomic dysfunction was also present. CRS is a multifactorial disease; ANS dysfunction should be considered when patients with excessive extranasal symptoms respond poorly to medical or surgical treatment. In clinical practice, COMPASS 31 can reliably be used to select CRS patients for further detailed ANS testing. As nerve impairment cannot be easily remedied, and because no definitive treatment is yet available, autonomic dysfunction is usually treated by addres- 
sing the symptoms and the underlying disease.(38) However, early diagnosis and control of autonomic dysfunction can reduce symptoms and improve the quality of life of CRS patients. A limitation of our study is that we could not determine the directional nature of the association between ANS dysfunction and CRS. ANS dysfunction may perpetuate CRS symptoms, or be a consequence of CRS, or bi-directional influences may be in play. The relationship between ANS dysfunction and CRS is very complex, and more work is needed. Further work involving ANS testing and exploring the impact of sinus surgery on ANS dysfunction is required.

\section{Conclusion}

We found a significant positive correlation between the symptom burden of ANS dysfunction and CRSwNP. The most common dysautonomia symptoms were excessively dry eyes, excessively dry mouth, postural dizziness, and a sensation of excessive fullness after a meal. Excessive coughing, post-nasal drip, sleep dysfunction, and psychological stress were closely associated with the severity of ANS dysfunction. In clinical practice, COMPASS 31 can be used to select patients who should undergo detailed ANS testing. Further research on the complex interrelationship of CRS and ANS dysfunction is warranted.

\section{Acknowledgements}

We appreciated the Biostatistics Centre, Kaohsiung Chang Gung Memorial Hospital for statistics work. This study was funded by grants obtained by Dr Wei-Chih Chen from Kaohsiung Chang Gung Memorial Hospital Taiwan (grant numbers CMRPG8E1511).

\section{Authorship contribution}

WCC: data collection, drafting this article

YTC: data analysis and interpretation

SFC: data analysis and interpretation

WCL: critical revision of the article

YYS: data collection and analysis

SDL: design of the study, critical revision of this article

\section{Conflict of interest}

None.

\section{References}

1. Barnes PJ. Neural control of human airways in health and disease. Am Rev Respir Dis 1986; 134:1289-1314.

2. Ricci A, Mariotta S, Amenta F, Tayebati SK, Terzano C. Changes in muscarinic cholinergic receptor expression in human peripheral blood lymphocytes in allergic rhinitis patients. Pulm Pharmacol Ther 2008; 21:7987.

3. Verma AR, Khodiar PK. Alteration of autonomic function is associated with disease severity in patients with bronchial asthma. Int J Clin Exp Physiol 2015; 2:110-114.

4. Williams HL. A concept of allergy as autonomic dysfunction suggested as an improved working hypothesis. Trans Am Acad Ophthalmol Otolaryngol 1950; 55:123-146.

5. Jaradeh SS, Smith TL, Torrico L, et al. Autonomic nervous system evaluation of patients with vasomotor rhinitis. Laryngoscope 2000; 110:1828-1831.

6. Loehrl TA, Smith TL, Darling RJ, et al. Autonomic dysfunction, vasomotor rhinitis, and extraesophageal manifestations of gastroesophageal reflux. Otolaryngol Head Neck Surg 2002; 126:382-387

7. Emin O, Esra G, Ufuk E, Demiri A, Ayhan S, Rusen DM. Autonomic dysfunction and clinical severity of disease in children with allergic rhinitis. Int J Pediatr Otorhinolaryngol 2012; 76:1196-1200.

8. Ozsutcu M, Ozkaya E, Demir A, Erenberk U, Sogut A, Dundaroz R. Pupillometric assessment of autonomic nervous system in children with allergic rhinitis. Med Princ Pract 2013; 22:444-448

9. Cerejeira R, Fernandes S, Pinto Moura C.
Increased expression of alpha7nAChR in chronic rhinosinusitis: The intranasal cholinergic anti-inflammatory hypothesis. Auris Nasus Larynx 2016; 43:176-181.

10. Low PA. Testing the autonomic nervous system. Semin Neurol 2003; 23:407-421.

11. Suarez GA, Opfer-Gehrking TL, Offord KP, Atkinson EJ, O'Brien PC, Low PA. The Autonomic Symptom Profile: a new instrument to assess autonomic symptoms. Neurology 1999; 52:523-528.

12. Sletten DM, Suarez GA, Low PA, Mandrekar J, Singer W. COMPASS 31: a refined and abbreviated Composite Autonomic Symptom Score. Mayo Clin Proc 2012; 87:1196-1201

13. Treister R, O'Neil K, Downs HM, Oaklander AL. Validation of the composite autonomic symptom scale 31 (COMPASS-31) in patients with and without small fiber polyneuropathy. Eur J Neurol 2015; 22:11241130.

14. Cortez MM, Nagi Reddy SK, Goodman B, Carter JL, Wingerchuk DM. Autonomic symptom burden is associated with MS-related fatigue and quality of life. Mult Scler Relat Disord 2015; 4:258-263.

15. Rosenfeld RM, Piccirillo JF, Chandrasekhar SS, et al. Clinical practice guideline (update): adult sinusitis. Otolaryngol Head Neck Surg 2015; 152:S1-S39.

16. Fokkens WJ, Lund VJ, Mullol J, et al European Position Paper on Rhinosinusitis and Nasal Polyps 2012. Rhinol Suppl 2012; 23:3 p preceding table of contents, 1-298.

17. Hopkins C, Gillett S, Slack R, Lund VJ, Browne JP. Psychometric validity of the 22-item Sinonasal Outcome Test. Clin Otolaryngol 2009; 34:447-454
18. DeConde AS, Mace JC, Bodner T, et al. SNOT-22 quality of life domains differentially predict treatment modality selection in chronic rhinosinusitis. Int Forum Allergy Rhinol 2014; 4:972-979.

19. Belafsky PC, Postma GN, Koufman JA. Validity and reliability of the reflux symptom index (RSI). J Voice 2002; 16:274-277.

20. Lund VJ, Mackay IS. Staging in rhinosinusitus. Rhinology 1993; 31:183-184.

21. Psaltis AJ, Li G, Vaezeafshar R, Cho KS Hwang PH. Modification of the LundKennedy endoscopic scoring system improves its reliability and correlation with patient-reported outcome measures. Laryngoscope 2014; 124:2216-2223.

22. Jartti T. Asthma, asthma medication and autonomic nervous system dysfunction. Clin Physiol 2001; 21:260-269.

23. Kim MH, Choi EJ, Jang BH, Kim KS, Ko SG, Choi I. Autonomic function in adults with allergic rhinitis and its association with disease severity and duration. Ann Allergy Asthma Immunol 2017; 118:174-178.

24. Ishman SL, Martin TJ, Hambrook DW, Smith TL, Jaradeh SS, Loehrl TA. Autonomic nervous system evaluation in allergic rhinitis. Otolaryngol Head Neck Surg 2007; 136:5156.

25. Rea NA, Campbell CL, Cortez MM. Quantitative assessment of autonomic symptom burden in Postural tachycardia syndrome (POTS). J Neurol Sci 2017; 377:3541.

26. Vieira B, Costa A, Videira G, Sa MJ, Abreu P. Prevalence of autonomic dysfunction in patients with multiple sclerosis. Acta Med Port 2015; 28:51-55.

27. Haid T. Vertigo originating from inflamma- 
tion of the paranasal sinuses (the so-called sinugenic vertigo). Adv Otorhinolaryngo 1981; 27:190-197.

28. Baroody FM. How nasal function influences the eyes, ears, sinuses, and lungs. Proc Am Thorac Soc 2011; 8:53-61.

29. Lester S, Rischmueller M, Tan L, et al. Sicca Symptoms and their Association with Chronic Rhinosinusitis in a Community Sample. Open Rheumatol J 2012; 6:170-174.

30. Leason SR, Barham HP, Oakley G, et al. Association of gastro-oesophageal reflux and chronic rhinosinusitis: systematic review and meta-analysis. Rhinology 2017; 55:3-16.

31. Katle EJ, Hatlebakk JG, Grimstad T, Kvaloy JT, Steinsvag SK. Gastro-oesophageal reflux in patients with chronic rhino-sinusitis investigated with multichannel impedance - $\mathrm{pH}$ monitoring. Rhinology 2017; 55:27-33.

32. Milovanovic B, Filipovic B, Mutavdzin S, et al. Cardiac autonomic dysfunction in patients with gastroesophageal reflux disease. World J Gastroenterol 2015; 21:6982-6989.

33. Lodi U, Harding SM, Coghlan HC, Guzzo MR, Walker LH. Autonomic regulation in asthmatics with gastroesophageal reflux. Chest 1997; 111:65-70.

34. Bucca C, Rolla G, Scappaticci E, et al. Extrathoracic and intrathoracic airway responsiveness in sinusitis. J Allergy Clin Immunol 1995; 95:52-59.

35. Alvares GA, Quintana DS, Hickie IB, Guastella AJ. Autonomic nervous system dysfunction in psychiatric disorders and the impact of psychotropic medications: a systematic review and meta-analysis. J Psychiatry Neurosci 2016; 41:89-104.

36. Chalmers JA, Quintana DS, Abbott MJ, Kemp AH. Anxiety Disorders are Associated with Reduced Heart Rate Variability: A Meta-Analysis. Front Psychiatry 2014; 5:80.

37. Miglis MG. Autonomic dysfunction in primary sleep disorders. Sleep Med 2016;
19:40-49.

38. McDougall AJ, McLeod JG. Autonomic neuropathy, I. Clinical features, investigation, pathophysiology, and treatment. J Neurol Sci 1996; 137:79-88.

\section{Sheng-Dean Luo}

Department of Otolaryngology

Kaohsiung Chang Gung Memorial

Hospital

No.123, Dapi Rd., Niaosong Dist.

Kaohsiung City 833

Taiwan

Tel: +886-7-7317123 ext. 2533;

Fax: +886-7-7313855

E-mail: rsd0323@cgmh.org.tw 